\title{
Potential dominated scalar-tensor cosmologies in the general relativity limit: phase space view
}

\author{
Laur Järv* Piret Kuusk ${ }^{\dagger}$ \\ Institute of Physics, University of Tartu, Riia 142, Tartu 51014, Estonia \\ Margus Saal ${ }^{\ddagger}$ \\ Tartu Observatory, Tõravere 61602, Estonia
}

\begin{abstract}
We consider the potential dominated era of Friedmann-Lemaitre-RobertsonWalker flat cosmological models in the framework of general Jordan frame scalartensor theories of gravity with arbitrary coupling functions, and focus upon the phase space of the scalar field. To study the regime suggested by the local weak field tests (i.e. close to the so-called limit of general relativity) we propose a nonlinear approximation scheme, solve for the phase trajectories, and provide a complete classification of possible phase portraits. We argue that the topology of trajectories in the nonlinear approximation is representative of those of the full system, and thus can tell for which scalar-tensor models general relativity functions as an attractor.
\end{abstract}

\section{Introduction}

The unknown source of observed present day acceleration of the Universe, called dark energy, is inspiring thorough investigations of different extensions of general relativity (GR) and $\Lambda \mathrm{CDM}$ cosmology (for recent reviews see Refs. [1]). The scalar-tensor theory of gravity (STG) [2] offers one such consistent possibility. Besides the usual spacetime metric tensor $g_{\mu \nu}$ it employs a scalar field $\Psi$, playing the role of a variable gravitational "constant", to describe the gravitational interaction. In the Jordan frame STG is specified by two functions [3], e.g. a coupling $\omega(\Psi)$ and a scalar potential $V(\Psi)$. In fact, a wide class of theories of gravitation, including higher order theories [4], theories of variable speed of light [5], as well as low energy approximations of brane world models and string theories [6] can be cast into the general form of STG.

\footnotetext{
*laur.jarv@ut.ee

†piret@fi.tartu.ee

${ }^{\ddagger}$ margus@fi.tartu.ee
} 
The weak field tests [7] pose a restriction to all alternative models of gravity including STG, since the Universe around us tends to be described by the Einstein tensorial gravity very precisely [8. This means that only those STG models are physically viable which in their late time cosmological evolution imply local consequences very close to those of GR. Several authors have studied how general relativity acts as an attractor for a wide class of STGs, such that the Solar System weak field (PPN) constraints spontaneously come to be satisfied at late times [9, 10, 11]. In our recent papers [12, 13] we have proposed a limiting process for the scalar field which describes scalar-tensor cosmological models relaxing to satisfy the Solar System constraints, with an indication for which classes of STGs the attractor behavior is realized.

The methods of dynamical systems have proved to be a useful tool when explicit analytic solutions are hard to find. In STG cosmology several authors have performed the analysis for different specific choices of the coupling and potential [14, while Refs. [15, 16, 17, 12] study the phase space and dynamics in the general case.

The STG phase space point corresponding to the limit of GR is peculiar in the sense that the standard linearization process there is hampered by ratios which turn out to be indeterminate. In our previous studies [12, 13, we assumed that these indeterminate terms vanish, which allows to treat this singular point as a standard fixed point. In the present paper we focus upon the potential dominated era of Friedmann-LemaitreRobertson-Walker (FLRW) flat cosmological models in the phase space of the (decoupled) scalar field $\Psi$ and its (cosmological) time derivative $\dot{\Psi} \equiv \Pi$, and propose an approximation which takes into account all possible finite values of these indeterminate ratios, thus preserving the leading nonlinear term in the field equations. We give a comprehensive description of the phase space trajectories of the approximate nonlinear system near the GR point and classify all trajectories allowed by the parameters of the theory. While the topology of trajectories differs in linear and nonlinear approximation, there is a correspondence in the final asymptotics, i.e. whether the trajectories end up at the GR point or are repelled from it. We argue that the nonlinear system accurately captures the key properties of the full system of STG equations near this singular point, and the topology of trajectories of the nonlinear approximation is representative of those of the full system. Therefore for any given STG model with a reasonable coupling $\omega(\Psi)$ and scalar potential $V(\Psi)$ our results predict whether GR is an attractor.

The paper is organized as follows. In the next section we review very briefly the scalar-tensor theory of gravity in the potential dominated era, write down the field equations of FLRW cosmology in the form of a dynamical system, and make some general remarks about the phase space including the singular GR point. In Sect. 3 we introduce the approximation method and present linear and nonlinear systems of equations in the neighbourhood of the singular point. In Section 4 we present solutions (phase trajectories) of the nonlinear system and a classification of trajectories, summarized in Table 1 and illustrated on Figure 1. In Section 5 our claims are backed up by a simple example. Finally, Section [ provides a summary and some remarks for future work. 


\section{Full equations and the phase space}

We consider a general scalar-tensor theory in the Jordan frame given by the action functional

$$
S=\frac{1}{2 \kappa^{2}} \int d^{4} x \sqrt{-g}\left[\Psi R(g)-\frac{\omega(\Psi)}{\Psi} \nabla^{\rho} \Psi \nabla_{\rho} \Psi-2 \kappa^{2} V(\Psi)\right] .
$$

(We have not included the matter contribution to the action, i.e. we consider only the potential dominated epoch.) Here $\omega(\Psi)$ is a coupling function (we assume $2 \omega(\Psi)+3 \geq 0$ to avoid ghosts in the Einstein frame, see e.g. Ref. [18]), $V(\Psi) \geq 0$ is a scalar potential, $\nabla_{\mu}$ denotes the covariant derivative with respect to the metric $g_{\mu \nu}$, and $\kappa^{2}$ is the nonvariable part of the effective gravitational constant $\frac{\kappa^{2}}{\Psi}$. In order to keep it positive we assume that $0<\Psi<\infty$.

The field equations for the flat Friedmann-Lemaître-Robertson-Walker (FLRW) line element

$$
d s^{2}=-d t^{2}+a(t)^{2}\left(d r^{2}+r^{2}\left(d \theta^{2}+\sin ^{2} \theta d \varphi^{2}\right)\right)
$$

read

$$
\begin{aligned}
H^{2} & =-H \frac{\dot{\Psi}}{\Psi}+\frac{1}{6} \frac{\dot{\Psi}^{2}}{\Psi^{2}} \omega(\Psi)+\frac{\kappa^{2}}{3} \frac{V(\Psi)}{\Psi} \\
2 \dot{H}+3 H^{2} & =-2 H \frac{\dot{\Psi}}{\Psi}-\frac{1}{2} \frac{\dot{\Psi}^{2}}{\Psi^{2}} \omega(\Psi)-\frac{\ddot{\Psi}}{\Psi}+\frac{\kappa^{2}}{\Psi} V(\Psi) \\
\ddot{\Psi} & =-3 H \dot{\Psi}-\frac{1}{2 \omega(\Psi)+3} \frac{d \omega(\Psi)}{d \Psi} \dot{\Psi}^{2}+\frac{2 \kappa^{2}}{2 \omega(\Psi)+3}\left[2 V(\Psi)-\Psi \frac{d V(\Psi)}{d \Psi}\right]
\end{aligned}
$$

where $H \equiv \dot{a} / a$.

By defining $\dot{\Psi} \equiv \Pi$, the equations (3)-(15) can be considered as an autonomous dynamical system, which is characterized by three variables $(\Psi, \Pi, H)$. However, one of them is algebraically related to the others via the Friedmann equation (3). We choose to

eliminate the Hubble parameter $H$ and thus reduce the system to two dimensions $(\Psi, \Pi)$. Upon introducing the functions

$$
A(\Psi) \equiv \frac{d}{d \Psi}\left(\frac{1}{2 \omega(\Psi)+3}\right), \quad W(\Psi) \equiv 2 \kappa^{2}\left(2 V(\Psi)-\frac{d V(\Psi)}{d \Psi} \Psi\right)
$$

the dynamical system reads

$$
\begin{aligned}
\dot{\Psi}= & \Pi, \\
\dot{\Pi}= & \left(\frac{3}{2 \Psi}+\frac{1}{2} A(\Psi)(2 \omega(\Psi)+3)\right) \Pi^{2} \\
& \pm \frac{1}{2 \Psi} \sqrt{3(2 \omega(\Psi)+3) \Pi^{2}+12 \kappa^{2} \Psi V(\Psi)} \Pi+\frac{W(\Psi)}{2 \omega(\Psi)+3} .
\end{aligned}
$$

Its regular phase trajectories and fixed points have been considered in Refs. [12, 17. 
Even if the scalar field rules the cosmological evolution, in the context of Solar System experiments we can reasonably assume that the energy density of the potential is negligible in comparison with the local matter density. Then the standard PPN analysis gives a condition for the present cosmological background value of the scalar field [7], (a) $\frac{1}{2 \omega(\Psi)+3} \rightarrow 0$. It is also evident that the effective gravitational constant is virtually immutable in time [10], which provides the second condition, (b) $\dot{\Psi} \equiv \Pi \rightarrow 0$. Let us denote $\Psi_{\star}$ the value of the scalar field where the coupling function $\omega(\Psi)$ has a singular "peak", or more precisely $\frac{1}{2 \omega\left(\Psi_{\star}\right)+3}=0$. Also, let $\Pi_{\star}$ be its vanishing time derivative, $\Pi_{\star}=0$. Then we may cautiously call the phase space point $\left(\Psi=\Psi_{\star}, \Pi=\Pi_{\star}\right)$ a 'GR point', since the STG solutions which can pass the local weak field tests and behave close enough to the ones of general relativity in terms of local observations, necessarily lie in the vicinity of this point.

The value $\Psi_{\star}$ poses a caveat, though. On the right hand side of Eq. (8) the $\left(2 \omega\left(\Psi_{\star}\right)+\right.$ 3) $\Pi^{2}$ terms are diverging if $\Pi \neq 0$ and indeterminate if $\Pi=\Pi_{\star}=0$. This means that the whole set $\Psi=\Psi_{\star}$ at arbitrary $\Pi$ is excluded from the (open) domain of definition of Eq. (8)). However, in what follows we find solutions of general approximated equations (Sect. 4) and of a specific full equation (Sect. 5) for phase trajectories which smoothly reach or pass through the point $\left(\Psi_{\star}, \Pi_{\star}\right)$. Thus we are justified to add the GR point to the open domain of definition as a boundary point. (Note that analogous conclusion can be inferred from our analysis of regions in the phase space accessible to phase trajectories in a general STG [12].)

The phase portrait of the dynamical system (7), (8) is drawn by the solutions (trajectories) of

$$
\begin{aligned}
\frac{d \Pi}{d \Psi}= & \left(\frac{3}{2 \Psi}+\frac{1}{2} A(\Psi)(2 \omega(\Psi)+3)\right) \Pi \\
& \pm \frac{1}{2 \Psi} \sqrt{3(2 \omega(\Psi)+3) \Pi^{2}+12 \kappa^{2} \Psi V(\Psi)}+\frac{W(\Psi)}{(2 \omega(\Psi)+3) \Pi}
\end{aligned}
$$

while the direction of the flow along them is determined by Eq. (7) as usual (towards increasing $\Psi$ for $\Pi>0$ and towards decreasing $\Psi$ for $\Pi<0$ ). Realizing that $\frac{d \Pi}{d \Psi}$ gives the slope of the tangent to a phase trajectory, a few characteristic features of the phase portrait outside the singular point $\left(\Psi_{\star}, \Pi_{\star}\right)$ can be immediately inferred.

First, on the horizontal line $\Pi=0$ ( $\Psi$-axis) the tangents of trajectories are vertically aligned if $W(\Psi) \neq 0$, since $\frac{d \Pi}{d \Psi}$ diverges due to the last term in Eq. (9). The inverted derivative $\left.\frac{d \Psi}{d \Pi}\right|_{\Pi=0}$ vanishes, while the sign of $\left.\frac{d^{2} \Psi}{d \Pi^{2}}\right|_{\Pi=0} \sim \frac{1}{W(\Psi)}$ indicates the direction of the flow along the trajectories: passing from $\Pi>0$ to $\Pi<0$ if $\left.\frac{d^{2} \Psi}{d \Pi^{2}}\right|_{\Pi=0}<0$ and vice versa if $\left.\frac{d^{2} \Psi}{d \Pi^{2}}\right|_{\Pi=0}>0$. The case of quadratic potential is special, as now $W(\Psi) \equiv 0$, and Eqs. (77), (8) reveal that besides the singular (indeterminate) point $\left(\Psi_{\star}, \Pi_{\star}\right)$, all points on the line $\Pi=0$ are fixed points, i.e. the trajectories do not pass through, but either begin or end there.

Second, approaching on the vertical line $\Psi=\Psi_{\star}$ the tangents of phase trajectories turn again vertical due to $(2 \omega(\Psi)+3)$ blowing up as $\Psi \rightarrow \Psi_{\star}$. As has been argued above, the line $\Psi=\Psi_{\star}(\Pi \neq 0)$ itself does not belong to the domain of the definition of the system, and here we see that the trajectories acknowledge this fact by not running 
into that line, but radically deflecting "up" or "down" instead, depending on the sign of $\left.\frac{d \Pi}{d \Psi}\right|_{\Psi \rightarrow \Psi_{\star}}$.

These two features qualitatively control the behavior of trajectories around the GR point. Consider for instance the region $\Psi<\Psi_{\star}, \Pi>0$ where the flow is directed towards $\Psi_{\star}$. First, if $\left.\frac{d^{2} \Psi}{d \Pi^{2}}\right|_{\Pi=0}<0$ at least some of these trajectories will cross over to the $\Pi<0$ belt and then flow away from $\Psi_{\star}$, effectively displaying a "saddle" type of behavior. On the other hand, if $\left.\frac{d^{2} \Psi}{d \Pi^{2}}\right|_{\Pi=0}>0$ there is no other option for the trajectories but to persist in flowing towards $\Psi_{\star}$, while additional trajectories coming over from the $\Pi<0$ region join their course. Second, near the $\Psi=\Psi_{\star}$ line if $\left.\frac{d \Pi}{d \Psi}\right|_{\Psi \rightarrow \Psi_{\star}}$ is positive, the flow is pushed "upwards" to $\Pi \rightarrow \infty$, while if $\left.\frac{d \Pi}{d \Psi}\right|_{\Psi \rightarrow \Psi_{\star}}$ is negative, the push is "downwards" towards smaller values of $\Pi$. In the latter case the outcome again depends on how the flow is directed on the $\Pi=0$ line, viz. if $\left.\frac{d^{2} \Psi}{d \Pi^{2}}\right|_{\Pi=0}<0$ the trajectories can cross over to the $\Pi<0$ region at any $\Psi \neq \Psi_{\star}$ and flow away, while if $\left.\frac{d^{2} \Psi}{d \Pi^{2}}\right|_{\Pi=0}>0$ the trajectories would have nowhere else to go but to hit the point $\left(\Psi_{\star}, \Pi_{\star}\right)$. A similar reasoning can be put forth for $\Psi>\Psi_{\star}$ as well. It may also happen that as the potential $V(\Psi)$ varies, the quantity $\left.\frac{d^{2} \Psi}{d \Pi^{2}}\right|_{\Pi=0} \sim \frac{1}{W(\Psi)}$ may be positive or negative depending on the value of $\Psi$ and the global picture gets rather complicated.

What can be said about the trajectories at the singular (indeterminate) point $\left(\Psi_{\star}\right.$, $\left.\Pi_{\star}\right)$ ? The logic of the phase space tells that passing through this point is possible from a region of the phase space where the flow is directed towards the point into a region of the phase space where the flow is directed away from the point. Under a reasonable assumption that (at least) the (physically relevant) solutions are continuous and smooth there are two such possibilities. First, the solutions may "slip through" $\Psi_{\star}$ from the region $\Psi<\Psi_{\star}, \Pi>0\left(\Psi>\Psi_{\star}, \Pi<0\right)$ to the region $\Psi>\Psi_{\star}, \Pi>0\left(\Psi<\Psi_{\star}, \Pi<0\right)$ if $\left.\frac{d \Pi}{d \Psi}\right|_{\Psi \rightarrow \Psi_{\star}, \Pi \rightarrow \Pi_{\star}}=0$, i.e. the tangent of the trajectory is aligned horizontally. Second, the solutions may "bounce back" from $\Psi_{\star}$ from the region $\Psi<\Psi_{\star}, \Pi>0\left(\Psi>\Psi_{\star}, \Pi<0\right)$ to the region $\Psi<\Psi_{\star}, \Pi<0\left(\Psi>\Psi_{\star}, \Pi>0\right)$ if $\left.\frac{d \Psi}{d \Pi}\right|_{\Psi \rightarrow \Psi_{\star}, \Pi \rightarrow \Pi_{\star}}=0$, i.e. the tangent of the trajectory is aligned vertically. Any trajectory hitting this point under a tangent which is neither horizontal nor vertical can not pass through the point, but must terminate there.

\section{Approximate equations}

The equations (77), (8) cannot be integrated without specifying the two arbitrary functions $\omega(\Psi)$ and $V(\Psi)$. But being interested in the behavior of solutions close to the GR point $\left(\Psi_{\star}, \Pi_{\star}\right)$ we can still proceed by considerning an approximation which maintains the key properties of the full system near this point. Although the full equations become singular (indeterminate) at $\left(\Psi_{\star}, \Pi_{\star}\right)$, we assume that the Taylor expansions of the functions $\omega(\Psi)$, $V(\Psi)$ are possible there.

Let us focus around the GR point,

$$
\Psi=\Psi_{\star}+x, \quad \Pi=\Pi_{\star}+y=y,
$$

where $x$ and $y$ span the neighbourhood of first order small distance from $\left(\Psi_{\star}, \Pi_{\star}\right)$. As phase space variables $x$ and $y$ are independent from each other, and so their ratio $y / x$ is indeterminate at $(x=0, y=0)$. The meaning of this indeterminacy is perhaps 
better illuminated in the polar coordinates $(\rho, \theta)$, where the radius $\rho$ is a first order small quantity, but $y / x \equiv \tan \theta \in(-\infty, \infty)$ becomes infinitely multivalued at the origin.

We can Taylor expand

$$
\frac{1}{2 \omega(\Psi)+3}=\frac{1}{2 \omega\left(\Psi_{\star}\right)+3}+A_{\star} x+\ldots \approx A_{\star} x
$$

and

$$
(2 \omega(\Psi)+3) \Pi^{2}=\frac{y^{2}}{0+A_{\star} x+\ldots}=\frac{y^{2}}{A_{\star} x}(1+O(x)) \approx \frac{y^{2}}{A_{\star} x},
$$

where $A_{\star} \equiv A\left(\Psi_{\star}\right)$. In order to keep the expansion under better control we have introduced here two additional conditions: (c) $A_{\star} \neq 0$, and $(\mathrm{d}) \frac{1}{2 \omega(\Psi)+3}$ is differentiable at $\Psi_{\star}$ ( $A_{\star}$ and higher derivatives do not diverge) [12, 13]. Although these assumptions somewhat constrain the possible forms of $\omega$, we are still dealing with a wide and relevant class of theories. In fact, the set (a)-(d) guarantees, that the second condition for the cosmological background value of the scalar field arising in the PPN analysis of STG [7], $\frac{1}{(2 \omega(\Psi)+3)^{3}} \frac{d \omega}{d \Psi} \rightarrow 0$, is automatically satisfied [12].

To simplify the notation, let us denote the values of some functions at $\left(\Psi_{\star}, \Pi_{\star}\right)$ as

$$
C_{1} \equiv \pm \sqrt{\frac{3 \kappa^{2} V\left(\Psi_{\star}\right)}{\Psi_{\star}}}, \quad C_{2} \equiv A_{\star} W_{\star},
$$

where $W_{\star} \equiv W\left(\Psi_{\star}\right)$ and $V\left(\Psi_{\star}\right) \geq 0$. The three constants $A_{\star}, W_{\star}, C_{1}$ determine the leading terms in expansions of the two functions $\omega(\Psi), V(\Psi)$ which specify a STG. Now the expansion of the solution for $H$ of the Friedmann constraint (3) reads

$$
\begin{aligned}
H & =-\frac{\Pi}{2 \Psi} \pm \sqrt{(2 \omega(\Psi)+3) \frac{\Pi^{2}}{12 \Psi^{2}}+\frac{\kappa^{2} V(\Psi)}{3 \Psi}} \\
& \approx-\frac{y}{2 \Psi_{\star}}+\frac{C_{1}}{3}\left[1+\frac{3 \kappa^{2}}{2 C_{1}^{2}} \frac{d}{d \Psi}\left(\frac{V}{\Psi}\right)_{\star} x+\frac{3}{8 C_{1}^{2} \Psi_{\star} A_{\star}} \frac{y^{2}}{x}+\ldots\right] .
\end{aligned}
$$

This explains the introduction of the \pm sign in the definition of $C_{1}$ in Eq. (13) , as near the GR point $(x=0, y=0)$ a positive constant, $C_{1}>0$, describes an expanding de Sitter Universe, while a negative one, $C_{1}<0$, describes a contracting de Sitter Universe.

Having outlined the method of approximation in the neighbourhood of $\left(\Psi_{\star}, \Pi_{\star}\right)$, let us apply it for the system (7), (8). If we assume, motivated by the condition (b), that physically relevant trajectories linger in the region close to the $x$-axis, i.e $\frac{y}{x}=\tan \theta$ being first order small, then in the expansion of Eq. (8) only the terms linear in $x$ and $y$ survive at the first order, while terms like $\frac{y^{2}}{x}$ (cf. Eq. (12)) can be dropped. This was the assumption implicit in our earlier analysis [12, 13]. In this case, denoting the variables $\tilde{x}$ and $\tilde{y}$, the approximation of (17), (8) yields a linear system

$$
\begin{aligned}
\dot{\tilde{x}} & =\tilde{y}, \\
\dot{\tilde{y}} & =C_{2} \tilde{x}-C_{1} \tilde{y},
\end{aligned}
$$

which, of course, is equivalent to a general second order linear homogeneous differential equation

$$
\ddot{\tilde{x}}+C_{1} \dot{\tilde{x}}-C_{2} \tilde{x}=0 \text {. }
$$


Its phase space analysis is well known, there is a fixed point at $(x=0, y=0)$ whose type depends on the values of the constants $C_{1}$ and $C_{2}$ (see e.g [19]).

However, in a more general case we must recognize the term $\frac{y^{2}}{x}$ as being the same order as $x$ and $y$. In other words, we consider all finite values of $\tan \theta$, and exclude only its infinite value on the $y$-axis which is outside the domain of definition of the system as said before. Thus, keeping the term $\frac{y^{2}}{x}$ in the approximation of (77), (8) , we obtain a nonlinear system

$$
\begin{aligned}
\dot{x} & =y, \\
\dot{y} & =\frac{y^{2}}{2 x}-C_{1} y+C_{2} x .
\end{aligned}
$$

The corresponding second order nonlinear differential equation reads

$$
\ddot{x}+C_{1} \dot{x}-C_{2} x=\frac{\dot{x}^{2}}{2 x} .
$$

Note that as distinct from eqs. (15), (16) , in this case point $(x=0, y=0)$ is not a fixed point with $\dot{x}=0, \dot{y}=0$ any more, but it is a singular point with an indeterminate and possibly multivalued term $\frac{y^{2}}{2 x}$ in eq. (19).

\section{Phase trajectories}

The phase trajectories for the nonlinear approximate system (18), (19) are determined by the equation

$$
\frac{d y}{d x}=\frac{y}{2 x}-C_{1}+\frac{x}{y} C_{2} .
$$

Its solutions

$$
|x| K=\left|\frac{1}{2} y^{2}+C_{1} y x-C_{2} x^{2}\right| \exp \left(-C_{1} f(u)\right), \quad u \equiv \frac{y}{x},
$$

depend on the sign of the expression $C_{1}^{2}+2 C_{2} \equiv C$, as the function $f(u)$ is given by

$$
\begin{array}{rlrl}
f(u) & =\frac{1}{\sqrt{C}} \ln \left|\frac{u+C_{1}-\sqrt{C}}{u+C_{1}+\sqrt{C}}\right| & \text { if } \quad C>0, \\
& =-\frac{2}{u+C_{1}} \quad \text { if } \quad C=0 \\
& =\frac{2}{\sqrt{|C|}}\left(\arctan \frac{u+C_{1}}{\sqrt{|C|}+n \pi)} \quad \text { if } \quad C<0 .\right.
\end{array}
$$

Here $K$ is a constant of integration which identifies the trajectory according to initial data $\left(x_{0}, y_{0}\right)$. Note that if we choose initial conditions from the allowed region $(\tan \theta$ is

finite), then our premise $\frac{y^{2}}{x} \sim y$ at deriving approximate equations (18), (19) is always valid, i.e. we get a small constant of integration, $K<1$.

In general, the right hand side of Eq. (21) can be written as a quotient of two second order homogeneous polynomials; a qualitative classification of the solutions of differential 
equations of this type was given by Lyagina [20] long time ago. In a nutshell, the phase portraits for different values of the constants $C_{1}$ and $C_{2}$ classify according to the number of sectors which form on the phase space around the origin $(x=0, y=0)$, and the topology of trajectories which inhabit these sectors. The sectors are separated by the boundary $x=0$ and invariant directions. The latter are lines $y=k x$ where the constant $k$ is a real solution of an algebraic equation

$$
k=\frac{k}{2}-C_{1}+\frac{C_{2}}{k},
$$

i.e straight trajectories $y=\left(-C_{1} \pm \sqrt{C}\right) x$ satisfying (21). All possible options are listed in Table 1 and graphically depicted on Figure 1.

If $C>0$ and $C_{2} \neq 0$ three directions divide the phase space into six topologically distinct sectors. The sectors can be elliptic where all trajectories start from the origin and get back to the origin, hyperbolic where all trajectories flow towards the origin but turn back before reaching it, or parabolic where all trajectories either start from afar and flow to the origin (stable case) or start from the origin and flow away (unstable case).

If $C_{1} \neq 0$ and $C_{2}=0$, i.e. the potential has a special form $V\left(\Psi_{\star}\right) \neq 0$, $\left(2 V\left(\Psi_{\star}\right)-\left.\frac{d V(\Psi)}{d \Psi}\right|_{\Psi_{\star}} \Psi_{\star}\right)=0$, then it follows from the original dynamical system (18), (19) that the entire $x$-axis (the point $x=0$ excluded) is populated by degenerate fixed points. There are four sectors. The two sectors which contain the $x$-axis are special, and do not properly belong to neither elliptic, hyperbolic or parabolic class, as the flow there is dominated by the cohort of fixed points lying on the $x$-axis. Let us provisionally call these 'sectors of degenerate fixed points'.

If $C=0$ there are four sectors. If both $C_{1}=0$ and $C_{2}=0$, the $x$-axis consists again of fixed points, while the generic trajectories are parabolas $2 K|x|=y^{2}$. If $C<0$ there are no real solutions to Eq. (27) and all we get are two elliptic sectors on both sides of the $y$-axis.

It is worth pointing out here, that the phase portraits of the nonlinear approximation display the same basic characteristic features we inferred about the solutions of the full system (9) before. First, on the horizontal axis $(y=0)$ the tangents of the trajectories are vertically aligned if $C_{2} \neq 0$, and the direction of the flow across $y=0$ is determined by the sign of $\left.\frac{d^{2} x}{d y^{2}}\right|_{y=0}$. If $C_{2}=0$ the horizontal axis is populated by fixed points. Second, next to the vertical axis $(x=0)$ the trajectories turn vertical and do not cross or intersect with the $x=0, y \neq 0$ line, deemed to be outside of the domain of definition of the system.

What happens at the origin $(x=0, y=0)$ where the sectors meet, needs an extra consideration. Inspection of the phase portraits on Figure 1 shows that in all cases there are multiple trajectories (identified by different values of $K$ ) which all reach the point in question. Although our solutions for the trajectories, Eq. (22), are given in terms of the phase space variables only and do not include time as an explicit parameter, the considerations presented in the end of Sect. 2 allow to draw some qualitative conclusions. First, there was a logical possibility of trajectories "slipping through" the origin so that $x$ changes its sign along a trajectory. It is evident from the phase portraits that this option is not realized in any of the cases, as none of the trajectories has $\tan \theta=0$ at this point. On the other hand, the second possibility, where the trajectories could "bounce back" from the origin so that $y$ changes its sign along a trajectory, is common to all cases, for 
there is always a class of trajectories whose tangent is vertically aligned at this point. Despite the fact that there seems to be loss of predictability here (the initial condition $x_{0}=0, y_{0}=0$ does not fix the constant $K$ uniquely), it would be natural to continue all such trajectories through this point keeping the same $K$ along them. Finally, those trajectories which reach the origin under finite $\tan \theta$ must either begin or end their flow at this point, like it happens at a regular fixed point.

It is also instructive to compare the phase portraits in the linear and nonlinear approximations. In the linear approximation (15), (16) the phase diagram is determined by

$$
\frac{d \tilde{y}}{d \tilde{x}}=-C_{1}+\frac{\tilde{x}}{\tilde{y}} C_{2}
$$

Its solutions are

$$
\tilde{K}=\left|\tilde{y}^{2}+C_{1} \tilde{x} \tilde{y}-C_{2} \tilde{x}^{2}\right| \exp \left(-\frac{C_{1}}{2} f(\tilde{u})\right), \quad \tilde{u} \equiv \frac{2 \tilde{y}}{\tilde{x}}
$$

where $f(\tilde{u})$ is given by the same expression (23), while instead of $C$ the solution is set by the sign of the expression $\tilde{C} \equiv C_{1}^{2}+4 C_{2}$. Now the invariant sectors are separated by the lines $\tilde{y}=\tilde{k} \tilde{x}$, where the constant $\tilde{k}$ is a real solution of

$$
\tilde{k}=-C_{1}+\frac{C_{2}}{\tilde{k}}
$$

i.e. $\tilde{k}=\frac{1}{2}\left(-C_{1} \pm \sqrt{\tilde{C}}\right)$. There is no indeterminacy in Eqs. (15), (16) and the point $(\tilde{x}=0, \tilde{y}=0)$ figures as a regular fixed point. The corresponding phase portraits in the neighbourhood of the origin can be classified according to a standard analysis [12, 13], the results being presented in Table 1.

The phase portraits in the nonlinear and linear approximation look markedly different, as in the linear case the line $\tilde{x}=0$ is not a boundary, and the trajectories for nodes, focuses etc. are not hindered from crossing it. Yet, modulo a factor of 2 in $C$ vs 4 in $\tilde{C}$, there is an overall correspondence in the classification, i.e. each distinct class in the linear case is matched with a distinct class in the nonlinear case (cf. Table 1).

This correspondence in the classification occurs due to an (accidental) property that the nonlinear system (18), (19) for $(x, y)$ can be formally obtained from the linear system (15), (16) for $(\tilde{x}, \tilde{y})$ by a replacement $C_{2} \rightarrow \frac{C_{2}}{2}$ (to get $\tilde{C} \rightarrow C$ ) and a simple transformation $\tilde{x}=\sqrt{|x|}, \tilde{y}=\frac{y}{2 \sqrt{|x|}}$. The latter can be understood as a mapping between the corresponding phase spaces. In particular, it squeezes the whole $\tilde{y}$-axis to a single point $(x=0, y=0)$, which manifests as the indeterminacy of the nonlinear system at this point. Another characteristic property of the map is the fact that the whole phase space $(\tilde{x}, \tilde{y})$ is mapped only on one half of the phase space $(x, y)$, but covering it twice. Since the transformation contains absolute value $|x|$, there are two separate images symmetric with respect to the $y$-axis.

Although the mapping between the linear and nonlinear systems seems to be only a mathematical coincidence and not a consequence of the fact that both the linear and nonlinear systems originate as approximations to the full system of equations (the nonlinear being a more general and refined one in this respect), we can nevertheless utilize this 
correspondece to unravel some useful information. Namely, the trajectories which cross the $\tilde{x}=0$ line at arbitrary $\tilde{y}$ in the linear case are mapped onto the trajectories which vertically hit the point $(x=0, y=0)$, and thus, as the former pass through the $\tilde{x}=0$ line the mapping suggests that also the latter must pass through the point $(x=0, y=0)$, hence supporting our reckoning above. Moreover, for $C<0$ or $\tilde{C}<0$ the trajectories depend not only on the real constant $K$ or $\tilde{K}$, but also on the integer $n$ due to the periodicity of arctan in $\mathrm{Eq}(23)$. Here in the linear case we have either a stable focus (spiralling trajectories flowing inwards into the origin) or unstable focus (spiralling trajectories flowing outwards from the origin), while $n$ decreases by 1 on each occasion when a trajectory flows through the $\tilde{y}$ axis. The mapping tells now, that also in the nonlinear case $n$ must decrease by 1 on each occasion when a trajectory flows through the point $(x=0, y=0)$, thus the overall picture being trajectories looping closer and closer or farther and farther from the origin. (On Figure 1 the diagrams 3.a and 3.c depict a trajectory with some fixed $K$ but different values of $n$, the direction of the flow is indicated by the increasing number of arrows on a trajectory loop.)

Thus (the mismatch of factor 2 vs 4 notwithstanding) besides the correspondence in the classification of the phase portraits, the linear and nonlinear approximations also share a qualitative correspondence in final asymptotic state of the flow, i.e. whether it ends up at the origin, or departs away from it. To summarize the results, it turns out that the GR point is an attractor for the asymptotic flow of all trajectories only if $C_{1}>0$ and $C_{2}<0$ (cases 1.c, 2.a, 3.a). If $C_{1}>0$ and $C_{2}=0$ all trajectories flow to the line $\Psi \neq \Psi_{\star}, \Pi=0$ instead (case 1.b). If $C_{1}=0$ and $C_{2}<0$ all trajectories loop through the GR point oscillating back and forth (nonlinear case 3.b), or if $C_{1}<0$ and $C_{2}<-\frac{C_{1}}{2}$ they oscillate further and further (nonlinear case 3.c). For the rest of the values of $C_{1}$ and $C_{2}$ all trajectories eventually flow away from the GR point.

\section{$5 \quad$ A special example}

To further illustrate how well the full system and its approximations near the GR point fit together, let us consider a STG with a specific coupling function and potential,

$$
\omega(\Psi)=\frac{3 \Psi}{2(1-\Psi)} \quad V(\Psi)=0 .
$$

From the condition $2 \omega+3 \geq 0$ it follows that $\Psi \leq 1$. The GR point is at $\Psi_{\star}=1, \Pi_{\star}=0$, while the rest of the border line, $\Psi_{\star}=1, \Pi_{\star} \neq 0$, is deemed to be outside of the definition of the system.

The full equation for phase trajectories, (9), reads now

$$
\frac{d \Pi}{d \Psi}=-\frac{3 \Pi}{2 \Psi}\left(-1 \pm \frac{1}{\sqrt{1-\Psi}}\right)-\frac{\Pi}{2(1-\Psi)}
$$

Fortunately it is amenable to integration, the solution is

$$
|\Pi|=K_{1} \sqrt{1-\Psi}(1 \pm \sqrt{1-\Psi})^{3},
$$

where $K_{1}$ is a constant of integration. 
No. Parameters Fixed point, Topology of trajectories, LS: $N=4 ; \mathrm{NLS}: N=2$ linear system nonlinear system

1. $C_{1}^{2}+N C_{2}>0$

1.a $C_{1}>0 \quad C_{2}>0$ saddle $\quad 2$ hyperb., 2 st. \& 2 unst. parab. sectors

1.b $\quad C_{1}>0 \quad C_{2}=0$ non-hyperbolic 1 stable \& 1 unstable parabolic sector, 2 stable sectors of degenerate fixed points

1.c $\quad C_{1}>0-\frac{C_{1}^{2}}{N}<C_{2}<0 \quad$ stable node $\quad 2$ elliptic, 4 stable parabolic sectors

1.d $C_{1}=0 \quad C_{2}>0$ saddle $\quad 2$ hyperb., 2 st. \& 2 unst. parab. sectors

1.e $C_{1}<0 \quad C_{2}>0$ saddle 2 hyperb., 2 st. \& 2 unst. parab. sectors

1.f $C_{1}<0 \quad C_{2}=0$ non-hyperbolic 1 stable \& 1 unstable parabolic sector, 2 unst. sectors of degenerate fixed points

1.g $\quad C_{1}<0-\frac{C_{1}^{2}}{N}<C_{2}<0 \quad$ unstable node $\quad 2$ elliptic, 4 unstable parabolic sectors

2. $C_{1}^{2}+N C_{2}=0$

2.a $\quad C_{1}>0 \quad C_{2}=-\frac{C_{1}^{2}}{N} \quad$ stable node $\quad 2$ elliptic, 2 stable parabolic sectors

2.b $C_{1}=0 \quad C_{2}=0 \quad$ free motion $\quad 2$ stable \& 2 unstable parabolic sectors

2.c $\quad C_{1}<0 \quad C_{2}=-\frac{C_{1}^{2}}{N} \quad$ unstable node $\quad 2$ elliptic, 2 unstable parabolic sectors

3. $C_{1}^{2}+N C_{2}<0$

3.a $\quad C_{1}>0 \quad C_{2}<-\frac{C_{1}^{2}}{N} \quad$ stable focus $\quad 2$ elliptic sectors

3.b $C_{1}=0 \quad C_{2}<0 \quad$ centre $\quad 2$ elliptic sectors

3.c $\quad C_{1}<0 \quad C_{2}<-\frac{C_{1}^{2}}{N} \quad$ unstable focus 2 elliptic sectors

Table 1: Types of fixed points for linear system (LS) and the topology of trajectories for nonlinear system (NLS). Definitions: $C=C_{1}^{2}+N C_{2}$, where $N=4$ for linear system and $N=2$ for nonlinear system. 


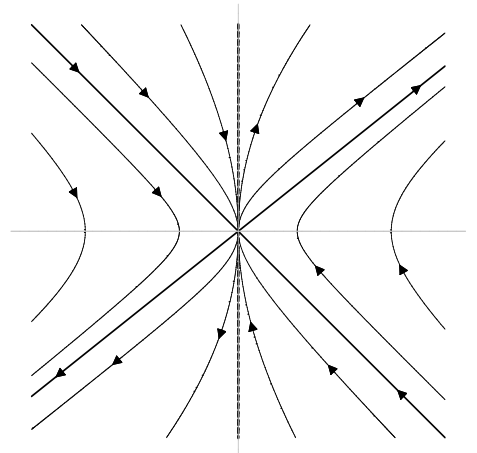

1.a (1.d)

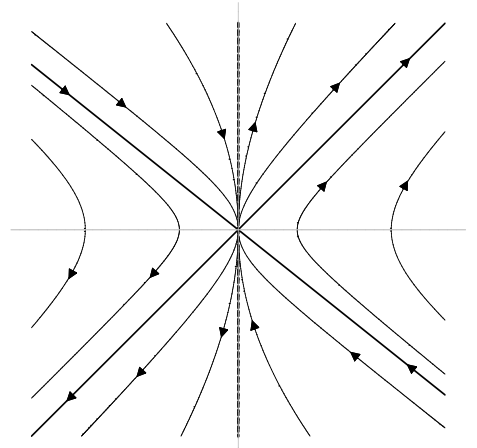

1.e (1.d)

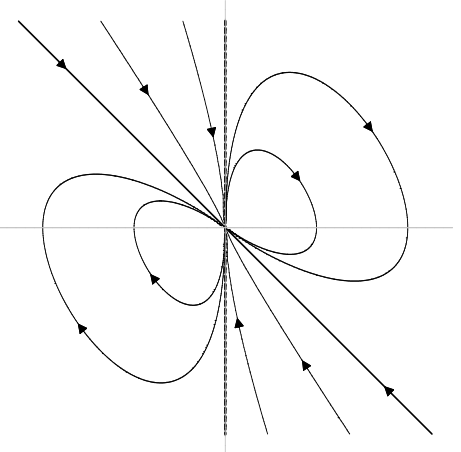

2.a

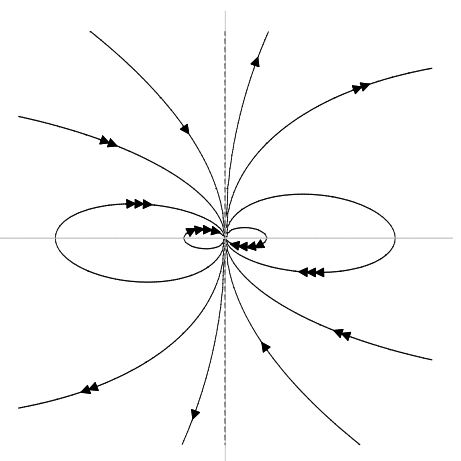

3.a

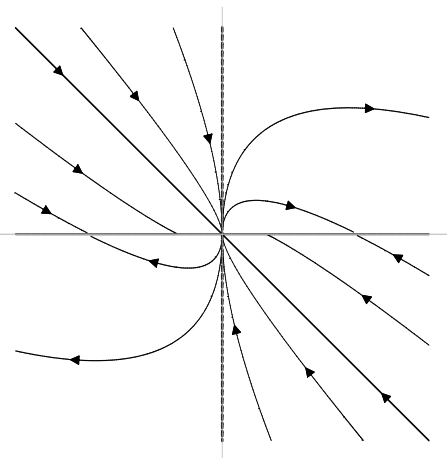

1.b

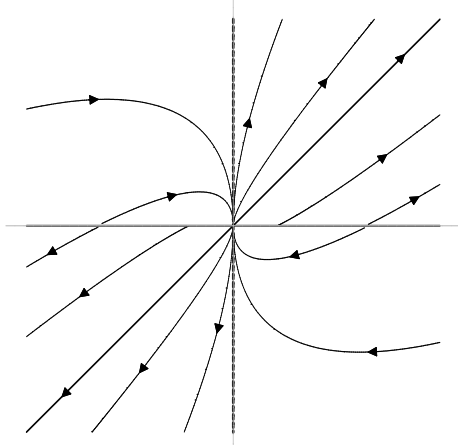

1.f

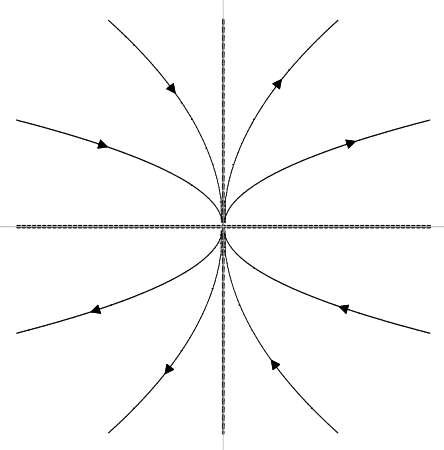

2.b

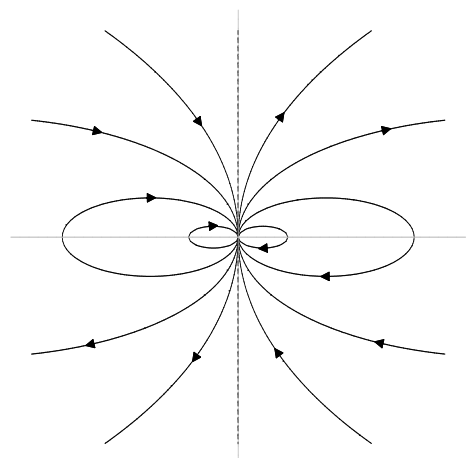

3.b

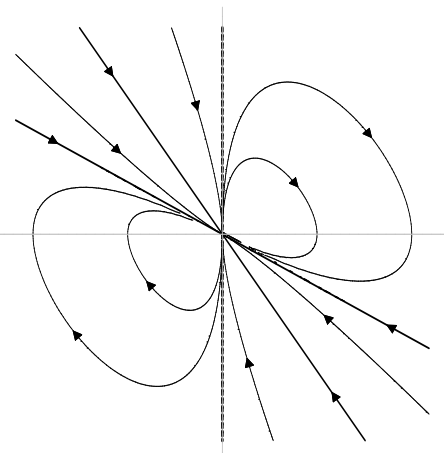

1.c

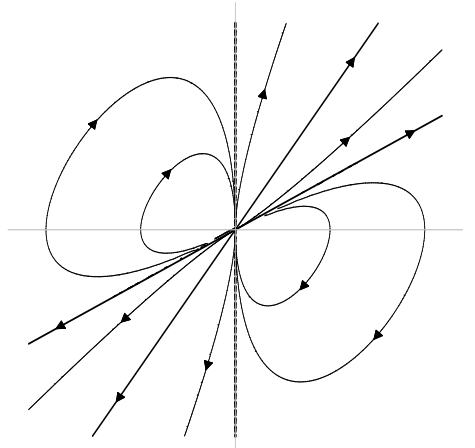

1.g

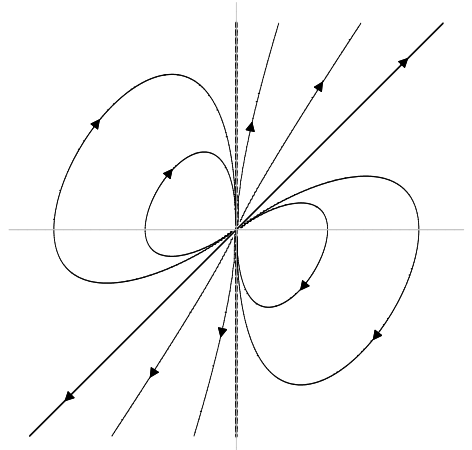

2.c

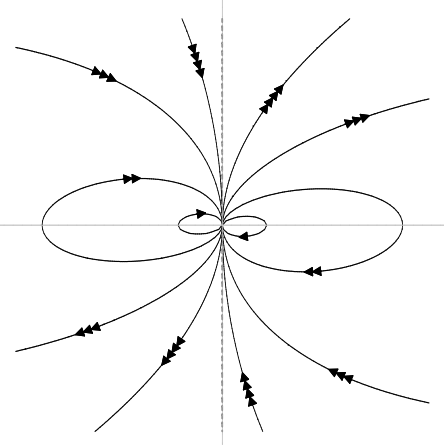

3.c

Figure 1: Phase portraits of the nonlinear approximation (21) near the GR point. 
Zooming to the vicinity of the GR point by (10), $1-\Psi=x>0, \Pi=y$, the solution (30) approximates to a parabola $|y|=K_{1} \sqrt{x}$, which by identifying the integration constants $K_{1}^{2}=2 K$ is in perfect accord with the solution $y^{2}=2 K x$ of the nonlinear approximate equation (21) with $C_{1}=0, C_{2}=0$ (case 2.b). This provides further evidence that the behavior of the full dynamical system (7), (8) near the GR point is adequately approximated by the nonlinear system (18), (19). Note also, that in the linear approximation we get a free motion, i.e. straight lines $\tilde{y}=\sqrt{\tilde{K}}$, which qualitatively gives a correct asymptotic state for the trajectories (flow away from the GR point), but clearly does not provide a faithful phase portrait in comparison with the full solution.

\section{Summary and Discussion}

This paper considers general scalar-tensor gravity (STG) in the Jordan frame with arbitrary coupling $\omega(\Psi)$ and potential $V(\Psi)$. We have presented and justified an approximate theory for the behavior of the scalar field in flat FLRW cosmological STG models in a potential dominated era and in the regime where the local weak field experiments are satisfied. In terms of the phase space $(\Psi, \dot{\Psi} \equiv \Pi)$ the latter is understood as the neighbourhood of the 'GR point' $\left(\Psi_{\star}, \Pi_{\star}\right)$, defined by (a) $\frac{1}{2 \omega\left(\Psi_{\star}\right)+3}=0$, (b) $\Pi_{\star}=0$. We propose

that if (c) $\frac{d}{d \Psi}\left(\frac{1}{2 \omega\left(\Psi_{\star}\right)+3}\right) \neq 0$ and (d) the higher derivatives of $\frac{1}{2 \omega\left(\Psi_{\star}\right)+3}$ do not diverge, then in the neighbourhood of the GR point the nonlinear system (18), (19) can be considered as an adequate approximate description of the full dynamical system (77), (8), since both are endowed with the same characteristic features. The phase portraits, summarized in Table 1 and depicted on Figure 1, typically show many trajectories passing through the GR point either once on multiple times. In the end, only if

$$
V\left(\Psi_{\star}\right)>0,\left.\left.\quad \frac{d}{d \Psi}\left(\frac{1}{2 \omega(\Psi)+3}\right)\right|_{\Psi_{\star}}\left(2 V(\Psi)-\frac{d V(\Psi)}{d \Psi} \Psi\right)\right|_{\Psi_{\star}}<0
$$

does the GR point function as an asymptotic attractor for the flow of all trajectories in the vicinity.

These analytic results could not have been predicted by numerical simulations, as the numerical calculations become rather problematic near the GR point due to the indeterminacy present in the equations. It would be very intersting to study how the different looping behaviors through the GR point manifest themselves in terms of observational predictions. It would also be of obvious physical relevance to extend the analysis to the matter dominated case, although the treatment of the problem would face a difficulty of having an additional phase space dimension to deal with.

\section{Acknowledgments}

This work was supported by the Estonian Science Foundation Grant No. 7185 and by Estonian Ministry for Education and Science Support Grant No. SF0180013s07. M.S. also acknowledges the Estonian Science Foundation Postdoctorial research Grant No. JD131. 


\section{References}

[1] E. J. Copeland, M. Sami and S. Tsujikawa, Int. J. Mod. Phys. D 15, 1753 (2006) arXiv:hep-th/0603057; J. Frieman, M. Turner and D. Huterer, Ann. Rev. Astron. Astrophys. 46, 385 (2008) [arXiv:0803.0982 [astro-ph]]; R. R. Caldwell and M. Kamionkowski, Ann. Rev. Nucl. Part. Sci. 59, 397 (2009) arXiv:0903.0866 [astroph.CO]].

[2] C. M. Will, Theory and experiment in gravitational physics, (Cambridge University Press, Cambridge, 1981); Y. Fujii and K. Maeda, The scalar-tensor theory of gravitation, (Cambridge University Press, Cambridge, 2003); V. Faraoni, Cosmology in scalar-tensor gravity, (Kluwer Academic Publishers, Dordrecht, 2004).

[3] E. E. Flanagan, Class. Quant. Grav. 21 (2004) 3817 arXiv:gr-qc/0403063.

[4] T. P. Sotiriou and V. Faraoni, arXiv:0805.1726 [gr-qc].

[5] J. Magueijo, Rept. Prog. Phys. 66, 2025 (2003) arXiv:astro-ph/0305457].

[6] M. Gasperini, String cosmology, (Cambridge University Press, Cambridge, 2007).

[7] K. J. Nordtvedt, Astrophys. J. 161, 1059 (1970); C. M. Will, Living Rev. Relativity 9 (2006) 3.

[8] G. Esposito-Farese, AIP Conf. Proc. 736, 35 (2004) arXiv:gr-qc/0409081; arXiv:gr-qc/0406001; Wei-Tou Ni, Int. J. Mod. Phys. D 14, 901 (2005), arXiv:gr-qc/0504116]; S. G. Turyshev, arXiv:0806.1731 [gr-qc]; B. Bertotti, L. Iess, and P. Tortora, Nature 425, 374 (2003); S. Tsujikawa, K. Uddin, S. Mizuno, R. Tavakol, and J. Yokoyama, Phys. Rev. D 77, 103009 (2008) arXiv:0803.1106 [astro-ph]].

[9] T. Damour and K. Nordtvedt, Phys. Rev. Lett. 70, 2217 (1993); T. Damour and K. Nordtvedt, Phys. Rev. D48, 3436 (1993).

[10] J. D. Barrow and P. Parsons, Phys. Rev. D 55, 1906 (1997) arXiv:gr-qc/9607072.

[11] J. D. Barrow and J. P. Mimoso, Phys. Rev. D 50, 3746 (1994); J.-M. Gérard and I. Mahara, Phys. Lett. B 346, 35 (1995); A. Serna and J. M. Alimi, Phys. Rev. D 53, 3074 (1996) arXiv:astro-ph/9510139; D. I. Santiago, D. Kalligas, and R. V. Wagoner, Phys. Rev. D 58, 124005 (1998), arXiv:gr-qc/9805044]; J. P. Mimoso and A. M. Nunes, Phys. Lett. A 248 (1998) 325; A. Navarro, A. Serna and J. M. Alimi, Phys. Rev. D 59 (1999) 124015 [arXiv:astro-ph/9903368]; A. Serna, J. M. Alimi, and A. Navarro, Class. Quant. Grav. 19, 857 (2002), |arXiv:gr-qc/0201049|; J. D. Barrow and D. J. Shaw, Class. Quant. Grav. 25, 085012 (2008) [arXiv:0712.2190 [gr-qc]]; C. E. M. Batista and W. Zimdahl, arXiv:0912.0998 [astro-ph.CO].

[12] L. Järv, P. Kuusk, and M. Saal, Phys. Rev. D 78, 083530 (2008) arXiv:0807.2159 $[\mathrm{gr}-\mathrm{qc}]]$. 
[13] P. Kuusk, L. Järv, and M. Saal, Int. Journ. Mod. Phys. A 24, 1631 (2009) arXiv:0810.5038[gr-qc]].

[14] E. Gunzig, V. Faraoni, A. Figueiredo, T. M. Rocha and L. Brenig, Class. Quant. Grav. 17, 1783 (2000); E. Gunzig, A. Saa, L. Brenig, V. Faraoni, T. M. Rocha Filho and A. Figueiredo, Phys. Rev. D 63, 067301 (2001) arXiv:gr-qc/0012085]; A. Saa, E. Gunzig, L. Brenig, V. Faraoni, T. M. Rocha Filho and A. Figueiredo, Int. J. Theor. Phys. 40, 2295 (2001) arXiv:gr-qc/0012105]; A. Figueiredo, T. M. Rocha Filho, E. Gunzig, L. Brenig and A. Saa, Int. J. Theor. Phys. 41, 2037 (2002) arXiv:gr-qc/0210090]; L. R. Abramo, L. Brenig, E. Gunzig and A. Saa, Int. J. Theor. Phys. 42, 1145 (2003) arXiv:gr-qc/0305008]; F. C. Carvalho and A. Saa, Phys. Rev. D 70087302 (2004) arXiv:astro-ph/0408013; L. Järv, P. Kuusk and M. Saal, Phys. Rev. D 75, 023505 (2007) |arXiv:gr-qc/0608109|; L. Järv, P. Kuusk, and M. Saal, Phys. Rev. D 76, 103506 (2007) [arXiv:0705.4644 [gr-qc]]; O. Hrycyna and M. Szydlowski, Phys. Rev. D 76, 123510 (2007) arXiv:0707.4471 [hep-th]]; K. i. Maeda and Y. Fujii, Phys. Rev. D 79, 084026 (2009) arXiv:0902.1221 [hep-th]].

[15] V. Faraoni, Ann. Phys. (N.Y.) 317, 366 (2005) [arXiv: gr-qc/0502015].

[16] A. Burd and A. Coley, Phys. Lett. B 267, 330 (1991).

[17] V. Faraoni, M. N. Jensen, and S. A. Theuerkauf, Class. Quant. Grav. 23, 4215 (2006) arXiv:gr-qc/0605050].

[18] G. Esposito-Farese and D. Polarski, Phys. Rev. D 63, 063504 (2001) arXiv:gr-qc/0009034.

[19] V. I. Arnold, Geometrical Methods in the Theory of Ordinary Differential Equations (Grundlehre der mathematischen Wissenschaften, vol. 250), 2nd edition, Springer, 1988.

[20] L. S. Lyagina, Uspekhi Mat. Nauk 6, 171 (1951). 